\title{
L-Quadratic Distribution
}

\author{
Salma Omar Bleed \\ College of Science \\ Department of Statistics \\ Al Asmarya University, Zlien Libya
}

\begin{abstract}
In this paper, the Generalization of the U-Quadratic Distribution using the quadratic rank transmutation map is developed called L-Quadratic $(\boldsymbol{L} \boldsymbol{Q})$ distribution with some important related integrations. Most of the mathematical properties are studied and the model parameters are estimated by the maximum likelihood method. Finally, an application to generated data sets is illustrated.
\end{abstract}

\section{Keywords}

U-Quadratic distribution, Transmutation Map, Maximum Likelihood Estimation, Survivor Function, Cumulative Hazard Function, Harmonic Mean, Moments, Moment Generating Function.

\section{INTRODUCTION}

The U-quadratic distribution is one of the types of continuous probability distributions with two parameters $\alpha$ and $\beta$. The distribution is often abbreviated $\boldsymbol{U} \boldsymbol{Q}(\boldsymbol{a}, \boldsymbol{b})$, and defined by the former two parameters as follows

$g(x)=\alpha(x-\beta)^{2}, \quad a<x<b, \quad a, b>0$

with distribution function (cdf)

$G(x)=\frac{\alpha}{3}\left[(x-\beta)^{3}+(\beta-a)^{3}\right], \quad a<x<b, \quad a, b>0$

where $\quad \alpha=\frac{12}{(b-a)^{3}} \quad$ (vertical scale), and $\quad \beta=\frac{b+a}{2}$ (gravitational balance center).

In applied probability theory, the $\boldsymbol{U} \boldsymbol{Q}$ distribution is one of the kinds of bimodal distributions. It is easily traceable to the modeling of symmetric bimodal processes with expected value and median : $\beta$, two Modes: $\mathrm{a}, \mathrm{b}$ and standard deviation: $0.387(b-a)$, [1].

In this paper, transmutation map approach suggested by Shaw and Buckley to define a new model which generalizes the $\boldsymbol{U} \boldsymbol{Q}$ model is used. It is called the generalized distribution as the L-Quadratic Distribution, because the pdf of the $\boldsymbol{L Q}$ distribution takes the form of the small letter " $l$ ", as shown in Figure (1). It is denoted by $\boldsymbol{L} \boldsymbol{Q}$ distribution and it is abbreviated $L Q(\mathbf{a}, \mathbf{b}, \boldsymbol{\lambda})$. In the rest of this paper, mathematical formulations with some important related integrations and properties of the $\boldsymbol{L} \boldsymbol{Q}$ distribution are provided, [2].

\section{L- QUADRATIC DISTRIBUTION}

According to the Quadratic Rank Transmutation Map (QRTM) approach, the cumulative distribution function (cdf) satisfy the relationship

$F(x)=(1+\lambda) G(x)-\lambda G^{2}(x)$

where $G(x)$ is the cumulative distribution function $(c d f)$ of the base distribution, which on differentiation yields, $f(x)$, such that $f(x)=(1+\lambda) g(x)-2 \lambda g(x) G(x)$

If $\lambda=0$ then the distribution of the base random variable is obtained. By using Eq.(2) and Eq.(3), the $c d f$ of $\boldsymbol{L Q}$ distribution has the following form

$F(x)=\frac{\alpha}{3}(x-\beta)^{3}\left[1-\frac{\alpha \lambda}{3}(x-\beta)^{3}\right]+\frac{1}{4}(\lambda+2)$

where $\lambda$ is the transmuted parameter. The corresponding pdf of Eq.(5) is given as follows

$f(x)=\alpha(x-\beta)^{2}\left[1-\frac{2 \alpha \lambda}{3}(x-\beta)^{3}\right]$

\section{STATISTICAL PROPERTIES}

Some statistical properties of the new generalization are provided, as follows, [3], [4]:

\subsection{Survivor Function}

There is a relation between the $c d f$ and the reliability function, i.e., $\boldsymbol{R} \boldsymbol{F}=\mathbf{1}-\boldsymbol{F}(\boldsymbol{x})$. Therefore, the Reliability Function (RF) of the $L Q$ distribution $\left(\boldsymbol{R} \boldsymbol{F}_{\boldsymbol{L Q}}\right)$ is defined as: $R F_{L Q}(x)=\frac{\alpha}{3}(x-\beta)^{3}\left[\frac{\alpha \lambda}{3}(x-\beta)^{3}-1\right]+\frac{1}{4}(2-\lambda)$

\subsection{Hazard Function}

There is a relation between the $p d f$, reliability and hazard function, i.e., $\boldsymbol{h}(\boldsymbol{x})=\frac{\boldsymbol{f}(\boldsymbol{x})}{\boldsymbol{R}(\boldsymbol{x})}$ Therefore, the Hazard Function (HF) of the $L Q$ distribution $\left(\boldsymbol{H} \boldsymbol{F}_{\mathbf{L Q}}\right)$ is defined as:

$$
H F_{L Q}(x)=\frac{\alpha(x-\beta)^{2}\left[1-\frac{2 \alpha \lambda}{3}(x-\beta)^{3}\right]}{\frac{\alpha}{3}(x-\beta)^{3}\left[\frac{\alpha \lambda}{3}(x-\beta)^{3}-1\right]+\frac{1}{4}(2-\lambda)}
$$

\subsection{Cumulative Hazard Function}

There is a relation between the $c d f$ and the cumulative hazard function, i.e., $\quad C H F(x)=-\ln F(x)$. Therefore, the Cumulative Hazard Function (CHF) of the $\boldsymbol{L} \boldsymbol{Q}$ distribution $\left(\boldsymbol{C H} \boldsymbol{F}_{\boldsymbol{L Q}}\right)$ is defined as :

$$
\boldsymbol{C H F}_{\boldsymbol{L} \boldsymbol{Q}}(x)=-\ln \left[\frac{\alpha}{3}(x-\beta)^{3}\left[1-\frac{\alpha \lambda}{3}(x-\beta)^{3}\right]+\frac{1}{4}(\lambda+2)\right] .
$$

As shown from Figure (1), the $\boldsymbol{L} \boldsymbol{Q}$ distribution is an extended model to analyze data from complex situations. Also, it is observed that, The pdf and the cumulative hazard function of the $\boldsymbol{L} \boldsymbol{Q}$ distribution takes the form of the small letter " $l$ ", but the cdf takes the form of the inverted " $\cap "$ quadratic distribution. The $\boldsymbol{L} \boldsymbol{Q}$ distribution has highest reliability at the lower limit "a", and then it is begins decreasing until the median, after that it is increasing again.

\subsection{Random Number Generation}

To generate random numbers when the parameters $a$ and $b$ are known, the method of inversion can be used from the $\boldsymbol{L} \boldsymbol{Q}$ distribution as

$X_{r v}=\beta+\left[\frac{3 u-0.75(2+\lambda)}{\alpha-0.5 \theta\left(X_{r v}-\beta\right)^{3}}\right]^{\frac{1}{3}}, \quad \theta=\frac{2 \alpha^{2} \lambda}{3}$ 
Eq.(7) doesn't have a closed form solution, so " $u$ " will be generated as uniform random variables from $U(0,1)$, and then solve for $X_{r v}$ in order to generate random numbers from $\boldsymbol{L Q}$ distribution. From Eq.(7), the quantile $X_{q}$ of the $\boldsymbol{L Q}$ distribution is given by

$X_{q}=\beta+\left[\frac{3 q-0.75(2+\lambda)}{\alpha-0.5 \theta\left(X_{q}-\beta\right)^{3}}\right]^{\frac{1}{3}}, \theta=\frac{2 \alpha^{2} \lambda}{3}$

Put $q=0.5$, the median of the $\boldsymbol{L} \boldsymbol{Q}$ distribution is obtained as $X_{0.5}=\beta+\left[\frac{0.75 \lambda}{0.5 \theta\left(X_{0.5}-\beta\right)^{3}-\alpha}\right]^{\frac{1}{3}}, \theta=\frac{2 \alpha^{2} \lambda}{3}$

Also, the percentiles and quartiles can be obtained, by putting different values of $q$ in Eq.(8), e.g., the $4^{\text {th }}$ quartiles, $90^{\text {th }}$ percentiles of the $\boldsymbol{L} \boldsymbol{Q}$ distribution is obtained when

$q=0.25, q=0.90$ respectively.

\subsection{Useful Important Integrations}

In this paper, the following integrations are developed by two forms for $r=1,2,3, \ldots$ as follows:

\section{The First Form (1):}

$A(1)=\int_{a}^{b} x^{r}(x-\beta)^{2} d x$

$=\sum_{j=0}^{2} \frac{(-1)^{j}\left(b^{r+j+1}-a^{r+j+1}\right)}{j !(r+j+1)} \cdot\left[\frac{\partial^{j}}{\partial \beta^{j}} \beta^{2}\right]$

$B(1)=\int_{a}^{b} x^{r}(x-\beta)^{5} d x$

$=\sum_{j=0}^{5} \frac{(-1)^{j+1}\left(b^{r+j+1}-a^{r+j+1}\right)}{j !(r+j+1)} \cdot\left[\frac{\partial^{j}}{\partial \beta^{j}} \beta^{5}\right]$

Proof

$A(1)=\int^{b} x^{r}(x-\beta)^{2} d x=\int^{b}\left\{x^{r+2}-2 \beta x^{r+1}+\beta^{2} x^{r}\right\} d x$ $=\frac{\left(b^{r+3}{ }^{a}-a^{r+3}\right)}{r+3}-2 \beta \frac{\left(b^{r+2}{ }^{a}-a^{r+2}\right)}{r+2}+\beta^{2} \frac{\left(b^{r+1}-a^{r+1}\right)}{r+1}$ $=\sum_{j=0}^{2} \frac{(-1)^{j}\left(b^{r+j+1}-a^{r+j+1}\right)}{j !(r+j+1)} \cdot\left[\frac{\partial^{j}}{\partial \beta^{j}} \beta^{2}\right]$

and

$B(1)=\int_{a}^{b} x^{r}(x-\beta)^{5} d x$

$=\int_{a}^{b}\left\{x^{r+5}-5 \beta x^{r+4}+10 \beta^{2} x^{r+3}-10 \beta^{3} x^{r+2}+5 \beta^{4} x^{r+1}\right.$

$\left.-\stackrel{a}{\beta^{5}} x^{r}\right\} d x$

$=\frac{\left(b^{r+6}-a^{r+6}\right)}{r+6}-5 \beta \frac{\left(b^{r+5}-a^{r+5}\right)}{r+5}+10 \beta^{2} \frac{\left(b^{r+4}-a^{r+4}\right)}{r+4}$

$-10 \beta^{3} \frac{\left(b^{r+3}-a^{r+3}\right)}{r+3}+5 \beta^{4} \frac{\left(b^{r+2}-a^{r+2}\right)}{r+2}$

$+\beta^{5} \frac{\left(b^{r+1}-a^{r+1}\right)}{r+1}$

$=\sum_{j=0}^{5} \frac{(-1)^{j+1}\left(b^{r+j+1}-a^{r+j+1}\right)}{j !(r+j+1)} \cdot\left[\frac{\partial^{j}}{\partial \beta^{j}} \beta^{5}\right]$

In general for $r=1,2,3, \ldots$

$$
\begin{aligned}
& \int_{a}^{b} x^{r}(x-\beta)^{s} d x \\
& = \begin{cases}\sum_{j=0}^{s} \frac{(-1)^{j}\left(b^{r+j+1}-a^{r+j+1}\right)}{j !(r+j+1)} \cdot\left[\frac{\partial^{j}}{\partial \beta^{j}} \beta^{s}\right], & s=0,2,4, \ldots \\
\sum_{j=0}^{s} \frac{(-1)^{j+1}\left(b^{r+j+1}-a^{r+j+1}\right)}{j !(r+j+1)} \cdot\left[\frac{\partial^{j}}{\partial \beta^{j}} \beta^{s}\right], & s=1,3,5, \ldots\end{cases}
\end{aligned}
$$

The Second Form (2):

$$
\begin{aligned}
& A(2)=\int_{a}^{b} x^{r}(x-\beta)^{2} d x \\
& =\sum_{j=0}^{2} \frac{(-1)^{j}\left(b^{r+j+1}-(-1)^{j} a^{r+j+1}\right)}{2^{2-j} \prod_{i=1}^{j+1}(r+i)} \cdot\left[\frac{\partial^{j}}{\partial Q^{j}} Q^{2}\right] \\
& B(2)=\int_{a}^{b} x^{r}(x-\beta)^{5} d x \\
& =\sum_{j=0}^{5} \frac{(-1)^{j}\left(b^{r+j+1}-(-1)^{j+1} a^{r+j+1}\right)}{2^{5-j} \prod_{i=1}^{j+1}(r+i)} \cdot\left[\frac{\partial^{j}}{\partial Q^{j}} Q^{5}\right]
\end{aligned}
$$

where $Q=(b-a)$

Proof: $A(2)=\int_{a}^{b} x^{r}(x-\beta)^{2} d x=\int_{a-\beta}^{b-\beta} y^{2}(y+\beta)^{r} d y$ using differentiation by parts, it is proved that

$$
\begin{aligned}
& A(2)=\int_{a}^{b} x^{r}(x-\beta)^{2} d x \\
& =\sum_{j=0}^{2} \frac{(-1)^{j}\left[b^{r+j+1}-(-1)^{j} a^{r+j+1}\right]}{2^{2-j} \prod_{i=1}^{j+1}(r+i)} \cdot\left[\frac{\partial^{j}}{\partial Q^{j}} Q^{2}\right]
\end{aligned}
$$

and

$$
\begin{aligned}
& B(2)=\int_{a}^{b} x^{r}(x-\beta)^{5} d x= \\
& \sum_{j=0}^{5} \frac{(-1)^{j}\left[b^{r+j+1}-(-1)^{j+1} a^{r+j+1}\right]}{2^{5-j} \prod_{i=1}^{j+1}(r+i)} \cdot\left[\frac{\partial^{j}}{\partial Q^{j}} Q^{5}\right]
\end{aligned}
$$

\begin{tabular}{|c|c|c|c|}
\hline \multicolumn{4}{|c|}{$\mathbf{A}(\mathbf{2})$} \\
\hline \multicolumn{2}{|c|}{$\begin{array}{l}n=25, a=1.038, b=7.274 \\
\lambda=0.151\end{array}$} & \multicolumn{2}{|c|}{$\begin{array}{l}n=30, a=1.326, b=7.428 \\
\lambda=0.100\end{array}$} \\
\hline $\mathrm{A}(1)$ & $\mathrm{A}(2)$ & $\mathrm{A}(1)$ & $\mathrm{A}(2)$ \\
\hline 83.998 & 83.998 & 82.889 & 82.889 \\
\hline 467.012 & 467.012 & 468.599 & 468.599 \\
\hline 2921.015 & 2921.015 & 2977.149 & 2977.149 \\
\hline 19068.861 & 19068.861 & 19814.846 & 19814.846 \\
\hline 126723.993 & 126723.993 & 134532.567 & 134532.567 \\
\hline 850219.148 & 850219.148 & 922883.188 & 922883.188 \\
\hline 5741058.791 & 5741058.791 & 6372979.704 & 6372979.704 \\
\hline 38961370.633 & 38961370.633 & 44228833.864 & 44228833.864 \\
\hline 265532964.483 & 265532964.483 & 308220461.918 & 308220461.918 \\
\hline
\end{tabular}

In general for $r=1,2,3, \ldots \quad, \quad \int_{a}^{b} x^{r}(x-\beta)^{s} d x=$ $\begin{cases}\sum_{j=0}^{s} \frac{(-1)^{j}\left(b^{r+j+1}-(-1)^{j} a^{r+j+1}\right)}{2^{s-j} \prod_{i=1}^{j+1}(r+i)} \cdot\left[\frac{\partial^{j}}{\partial Q^{j}} Q^{s}\right], & s=0,2,4, \ldots \\ \sum_{j=0}^{S} \frac{(-1)^{j}\left(b^{r+j+1}-(-1)^{j+1} a^{r+j+1}\right)}{2^{s-j} \prod_{i=1}^{j+1}(r+i)} \cdot\left[\frac{\partial^{j}}{\partial Q^{j}} Q^{s}\right], & s=1,3,5, \ldots\end{cases}$

For given samples with different choices of $a, \mathrm{~b}$ and $\lambda$, it is proved that, the integrations of Eq.9 and Eq.11 gave the same results, Table 1 summarizes the results.

Table 1. The Numerical Results of the integrations A(1) and A(2) 
Also, for given samples with different choices of $a$, b and $\lambda$, it is proved that, the integrations of Eq.10 and Eq. gave the same results, Table 2 summarizes the results

Table 2. The Numerical Results of the integrations $B(1)$ and $B(2)$

\begin{tabular}{|c|c|c|c|}
\hline \multicolumn{2}{|c|}{$\begin{array}{l}n=25, a=1.038, b=7.274 \\
\lambda=0.151\end{array}$} & \multicolumn{2}{|c|}{$\begin{array}{l}n=30, a=1.326, b=7.428 \\
\lambda=0.100\end{array}$} \\
\hline $\mathrm{B}(1)$ & $\mathrm{B}(2)$ & $\mathrm{B}(1)$ & $\mathrm{B}(2)$ \\
\hline 818.725 & 818.725 & 703.379 & 703.379 \\
\hline 6805.652 & 6805.652 & 6157.646 & 6157.646 \\
\hline 48620.070 & 48620.070 & 45522.784 & 45522.783 \\
\hline 338054.080 & 338054.080 & 325130.688 & 325130.684 \\
\hline 2340281.552 & 2340281.552 & 2305646.766 & 2305646.738 \\
\hline 16210654.402 & 16210654.402 & 16342813.696 & 16342813.506 \\
\hline 112473810.571 & 112473810.571 & 115981914.625 & 115981913.316 \\
\hline 781800350.335 & 781800350.335 & 824421395.565 & 824421386.519 \\
\hline 5443934588.051 & 5443934588.051 & 5869711034.899 & 5869710972.075 \\
\hline
\end{tabular}

\subsection{Central Tendency}

The mean, median, mode of the $\boldsymbol{L} \boldsymbol{Q}$ distribution can be obtained as follows:

Theorem 1: If $X$ a r.v has $\boldsymbol{L Q}$ distribution then the mean is: $\mu=\dot{\mu}_{1}=\beta-\frac{3 \lambda}{14}(b-a)$

Proof: By using the integrations of Eq.9 to Eq.12, the mean of the $\boldsymbol{L} \boldsymbol{Q}$ distribution is:

$\mu=\dot{\mu}_{1}=\int_{a}^{b} x f(x) d x=\int_{a}^{b} x\left[\alpha(x-\beta)^{2}-\theta(x-\beta)^{5}\right] d x$

Then,

$\mu=\mu(A)$

$=\sum_{j=0}^{2} \frac{(-1)^{j}\left(b^{j+2}-a^{j+2}\right)}{j !(j+2)} \cdot\left[\frac{\partial^{j}}{\partial \beta^{j}}\left(\alpha \beta^{2}\right)\right]$

$-\sum_{j=0}^{5} \frac{(-1)^{j+1}\left(b^{j+2}-a^{j+2}\right)}{j !(j+2)} \cdot\left[\frac{\partial^{j}}{\partial \beta^{j}}\left(\theta \beta^{5}\right)\right]$

$\mu=\mu(B)$

$=\alpha \sum_{j=0}^{2} \frac{(-1)^{j}\left(b^{j+2}-(-1)^{j} a^{j+2}\right)}{2^{2-j} \prod_{i=1}^{j+1}(1+i)} \cdot\left[\frac{\partial^{j}}{\partial Q^{j}} Q^{2}\right]$

$-\theta \sum_{j=0}^{5} \frac{(-1)^{j}\left(b^{j+2}-(-1)^{j+1} a^{j+2}\right)}{2^{5-j} \prod_{i=1}^{j+1}(1+i)} \cdot\left[\frac{\partial^{j}}{\partial Q^{j}} Q^{5}\right]$

So, $\mu=\mu(A)=\mu(B)=\beta-\frac{3 \lambda}{14}(b-a)$

For different choices of $a, \mathrm{~b}$ and $\lambda$, it is proved that, the forms of the arithmetic mean in Eq.13, Eq.14 and Eq.15 gave the same results, Table 3 summarizes the results.

Table 3. The Numerical Solution of the mean

\begin{tabular}{|l|l|l|l|l|l|}
\hline $\mathrm{a}$ & $\mathrm{b}$ & $\lambda$ & $\mu$ & $\mu(A)$ & $\mu(B)$ \\
\hline 0.05 & 0.10 & 0.50 & 0.0696 & 0.0696 & 0.0696 \\
\hline 0.05 & 0.20 & 0.75 & 0.1009 & 0.1009 & 0.1009 \\
\hline 0.10 & 1.00 & 1.00 & 0.3571 & 0.3571 & 0.3571 \\
\hline 0.25 & 1.50 & 1.50 & 0.4286 & 0.4286 & 0.4286 \\
\hline 0.50 & 1.50 & 2.00 & 0.5714 & 0.5714 & 0.5714 \\
\hline 1.00 & 2.00 & 2.50 & 1.1786 & 1.1786 & 1.1786 \\
\hline 2.00 & 3.00 & 2.75 & 1.9107 & 1.9107 & 1.9107 \\
\hline 2.50 & 3.25 & 3.00 & 2.3929 & 2.3929 & 2.3929 \\
\hline 3.00 & 4.00 & 3.50 & 2.7500 & 2.7500 & 2.7500 \\
\hline 5.00 & 10.0 & 6.00 & 1.0714 & 1.0714 & 1.0714 \\
\hline
\end{tabular}

Theorem 2: If $X$ a r.v has $\boldsymbol{L} \boldsymbol{Q}$ distribution then the median is: $m=\beta+\left[\frac{9 \lambda}{4 \alpha\left[\alpha \lambda(m-\beta)^{3}-3\right]}\right]^{1 / 3}$

Proof:

$$
\begin{aligned}
0.5=\int_{a}^{m} f(x) d x=\int_{a}^{m} & {\left[\alpha(x-\beta)^{2}-\theta(x-\beta)^{5}\right] d x } \\
& =\frac{\alpha}{3}(m-\beta)^{3}\left[1-\frac{\alpha \lambda}{3}(m-\beta)^{3}\right]+\left(\frac{\lambda+2}{4}\right)
\end{aligned}
$$

Then, $m=\beta+\left[\frac{9 \lambda}{4 \alpha\left[\alpha \lambda(m-\beta)^{3}-3\right]}\right]^{1 / 3}$ and it is equivalents of Eq.(8).

Theorem 3: If $X$ r.v has $\boldsymbol{L Q}$ distribution then the mode is: mode $=m o=\beta+\left[\frac{0.6}{\alpha \lambda}\right]^{1 / 3}$

Proof: By taking the $1^{\text {st }}$ derivative of Eq.(6) with respect to the r.v X,

$f(x)=2 \alpha(x-\beta)^{3}\left[1-\frac{5 \alpha \lambda}{3}(x-\beta)^{3}\right]$

Then, $m o=\beta+\left[\frac{0.6}{\alpha \lambda}\right]^{1 / 3}$, where

$\hat{f}(x)=2 \alpha-\frac{40 \alpha^{2} \lambda}{3}(x-\beta)^{3}<0$

Theorem 4: If $X$ a r.v has $\boldsymbol{L} \boldsymbol{Q}$ distribution then the Harmonic mean $(\mathrm{Hm})$ is:

$$
\begin{aligned}
& =\left\{\alpha \beta^{2}\left(1+\frac{2 \alpha \lambda \beta^{3}}{3}\right) \ln \left(\frac{b}{a}\right)-\alpha \beta(b-a)\right. \\
& \left.-\sum_{j=0}^{5} \frac{(-1)^{j+1}\left(b^{j}-a^{j}\right)}{j !(j)} \cdot\left[\frac{\partial^{j}}{\partial \beta^{j}}\left(\theta \beta^{5}\right)\right]\right\}^{-1}
\end{aligned}
$$

Proof :

$\frac{1}{H m}=E\left(\frac{1}{x}\right)=\int_{a}^{b} \frac{1}{x} f(x) d x=\int_{a}^{b} \frac{1}{x}\left[\alpha(x-\beta)^{2}-\right.$

$\left.{ }_{\theta}(x-\beta)^{5}\right] d x=\alpha \beta^{2}\left(1+\frac{2 \alpha \lambda \beta^{3}}{3}\right) \ln \left(\frac{b}{a}\right)-\alpha \beta(b-a)-$

$\sum_{j=0}^{5} \frac{(-1)^{j+1}\left(b^{j}-a^{j}\right)}{j !(j)} \cdot\left[\frac{\partial^{j}}{\partial \beta^{j}}\left(\theta \beta^{5}\right)\right]$

Then, $H m=\left\{\alpha \beta^{2}\left(1+\frac{2 \alpha \lambda \beta^{3}}{3}\right) \ln \left(\frac{b}{a}\right)-\alpha \beta(b-a)-\right.$ $\left.\sum_{j=0}^{5} \frac{(-1)^{j+1}\left(b^{j}-a^{j}\right)}{j !(j)} \cdot\left[\frac{\partial^{j}}{\partial \beta^{j}}\left(\theta \beta^{5}\right)\right]\right\}^{-1}$

\subsection{Moments}

Theorem 5: If $X$ a r.v has $\boldsymbol{L} \boldsymbol{Q}$ distribution then the $r^{\text {th }}$ moments are :

$$
\begin{aligned}
& \dot{\mu}_{r}=\mu\left(A_{r}\right) \\
& =\sum_{j=0}^{2} \frac{(-1)^{j}\left(b^{r+j+1}-a^{r+j+1}\right)}{j !(r+j+1)} \cdot\left[\frac{\partial^{j}}{\partial \beta^{j}}\left(\alpha \beta^{2}\right)\right] \\
& -\sum_{j=0}^{5} \frac{(-1)^{j}\left(b^{r+j+1}-a^{r+j+1}\right)}{j !(r+j+1)} \cdot\left[\frac{\partial^{j}}{\partial \beta^{j}}\left(\theta \beta^{5}\right)\right]
\end{aligned}
$$




$$
\begin{aligned}
& \dot{\mu}_{r}=\mu\left(B_{r}\right) \\
& =\alpha \sum_{j=0}^{2} \frac{(-1)^{j}\left(b^{r+j+1}-(-1)^{j} a^{r+j+1}\right)}{2^{s-j} \prod_{i=1}^{j+1}(r+i)} \cdot\left[\frac{\partial^{j}}{\partial Q^{j}} Q^{s}\right] \\
& -\theta \sum_{j=0}^{s} \frac{(-1)^{j}\left(b^{r+j+1}-(-1)^{j+1} a^{r+j+1}\right)}{2^{s-j} \prod_{i=1}^{j+1}(r+i)} \cdot\left[\frac{\partial^{j}}{\partial Q^{j}} Q^{s}\right]
\end{aligned}
$$

Proof: Using the integrations of Eq.9 to Eq.12, The $r^{\text {th }}$ ordinary moment of the $\boldsymbol{L} \boldsymbol{Q}$ distribution is given by : $\dot{\mu}_{r}=\int_{a}^{b} x^{r} f(x) d x=\int_{a}^{b} x^{r}\left[\alpha(x-\beta)^{2}-\theta(x-\beta)^{3}\right] d x$

Then,

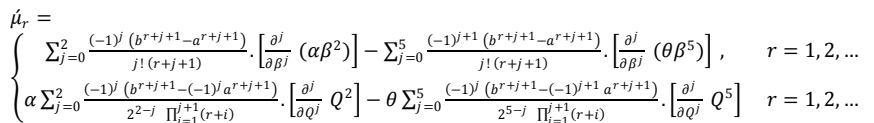

so,

$\dot{\mu}_{1}=\beta-\frac{3 \lambda}{14}(b-a), \quad \dot{\mu}_{2}=\frac{\left(b^{2}+a^{2}\right)}{2}-\frac{(b-a)^{2}}{10}-$

$\frac{3 \lambda\left(b^{2}-a^{2}\right)}{14}$

$\dot{\mu}_{3}=\frac{\left(b^{3}+a^{3}\right)}{2}-\frac{3(b+a)(b-a)^{2}}{20}-\frac{\lambda\left(b^{3}-a^{3}\right)}{4}+\frac{3 \lambda\left(b^{2}+a^{2}\right)(b-a)}{56}-$

$\frac{3 \lambda(b-a)^{3}}{7\left(2^{8}\right)}, \quad \sigma^{2}=\frac{3(b-a)^{2}}{4}\left[\frac{1}{5}-\frac{3 \lambda^{2}}{49}\right]$.

If $\lambda=0$ then the first three moments of the base random variable are obtained:

$\dot{\mu}_{1}=\beta=\mu, \quad \dot{\mu}_{2}=\frac{\left(b^{2}+a^{2}\right)}{2}-\frac{(b-a)^{2}}{10} \quad, \quad \dot{\mu}_{3}=\frac{\left(b^{3}+a^{3}\right)}{2}-$ $\frac{3(b+a)(b-a)^{2}}{20}$ and $\sigma^{2}=0.15(b-a)^{2}$

\subsection{Moment Generating Function}

The moment generating function (mgf) is important especially if it is existing. Then the moment generating function of $\boldsymbol{L} \boldsymbol{Q}$ distribution is derived.

Theorem 6: If $X$ a r.v has the $\boldsymbol{L} \boldsymbol{Q}$ distribution then the mgf is: $\boldsymbol{m} \boldsymbol{g} \boldsymbol{f}_{\boldsymbol{L Q}}=m_{x}(t)=\sum_{r=1}^{\infty} \frac{t^{r}}{r !} \dot{\mu}_{r}$

Proof:

$\boldsymbol{m} \boldsymbol{g} \boldsymbol{f}_{L \boldsymbol{Q}}=m_{x}(t)=E\left(e^{t x}\right)=\int_{a}^{b} e^{t x} f(x) d x=$ $\int_{a}^{b} e^{t x}\left[\alpha(x-\beta)^{2}-\theta(x-\beta)^{3}\right] d x$

Then

$\boldsymbol{m} \boldsymbol{g} \boldsymbol{f}_{\boldsymbol{L} \boldsymbol{Q}}=\sum_{r=1}^{\infty} \frac{t^{r}}{r !}\left[\int_{a}^{b}\left[\alpha x^{r}(x-\beta)^{2}-\theta x^{r}(x-\beta)^{3}\right] d x\right]=$ $\sum_{r=1}^{\infty} \frac{t^{r}}{r !} \dot{\mu}_{r}$

Put $t=i t$ in Eq.(15), then characteristic function $\left(\mathrm{Q}_{L Q}\right)$ of the $\boldsymbol{L} \boldsymbol{Q}$ distribution is:

$$
\begin{gathered}
\boldsymbol{Q}_{L Q}=\sum_{r=1}^{\infty} \frac{(i t)^{r}}{r !}\left[\int_{a}^{b}\left[\alpha x^{r}(x-\beta)^{2}-\theta x^{r}(x-\beta)^{3}\right] d x\right] \\
=\sum_{r=1}^{\infty} \frac{(i t)^{r}}{r !} \dot{\mu}_{r}
\end{gathered}
$$

From Eq.(15), notice that, the $\mathrm{r}^{\text {th }}$ moment is the coefficient of $\frac{t^{r}}{r !}$, i.e., $\dot{\mu}_{r}=$ coef.of $\frac{t^{r}}{r !}$, and if $\lambda=0$ then

$\boldsymbol{m g f}_{\boldsymbol{L Q D} \boldsymbol{D}}=\sum_{r=1}^{\infty} \frac{t^{r}}{r !}\left\{\int_{a}^{b}\left[\alpha x^{r}(x-\beta)^{2}\right] d x\right\}=\sum_{r=1}^{\infty} \frac{t^{r}}{r !} \dot{\mu}_{r}$ which is the mgf of the $\boldsymbol{U} \boldsymbol{Q}$ distribution.

\section{PARAMETER ESTIMATION}

The maximum likelihood estimates, MLEs, of the parameters that are inherent within the $\boldsymbol{L} \boldsymbol{Q}$ distribution function is given by the following: Let $x_{1}, x_{2}, \ldots, x_{n}$ be a sample of size $\mathrm{n}$ from $\boldsymbol{L} \boldsymbol{Q}$ distribution, then the likelihood function is given by

$L=\alpha^{n}\left\{\prod_{i=1}^{n}\left[\left(x_{i}-\beta\right)^{2}\left(1-\frac{2 \alpha \lambda}{3}\left(x_{i}-\beta\right)^{3}\right)\right]\right\}$

Put $w_{i}(\beta)=\left(x_{i}-\beta\right)$ and $w_{i}(\underline{\Phi})=\left(1-\frac{2 \alpha \lambda}{3} w_{i}^{3}(\beta)\right), \quad \underline{\Phi}=$ $(\alpha, \lambda, \beta)$

Then $L=\alpha^{n}\left\{\prod_{i=1}^{n} w_{i}^{2}(\beta) w_{i}(\underline{\Phi})\right\}$

The log-likelihood function of Eq.(16) is given by

$l=\ln L=n \ln \alpha+2 \sum_{i=1}^{n} \ln w_{i}(\beta)+\sum_{i=1}^{n} \ln w_{i}(\underline{\Phi})$

The log-likelihood can be maximized by differentiating Eq.(17) to obtain the maximum likelihood estimate $(M L E)$ of the unknown parameter $(\alpha, \lambda, \beta)$. Therefore, The $1^{\text {st }}$ partial derivatives of Eq.(17) with respect to the unknown parameters $(\alpha, \lambda, \beta)$ are given by:

$\frac{\partial l}{\partial \alpha}=\frac{n}{\alpha}-\frac{2 \lambda}{3} \sum_{i=1}^{n}\left[w_{i}^{3}(\beta) w_{i}^{-1}(\underline{\Phi})\right]$

$\frac{\partial l}{\partial \beta}=2 \sum_{i=1}^{n} w_{i}^{-1}(\beta)\left[\alpha \lambda w_{i}^{3}(\beta) w_{i}^{-1}(\underline{\Phi})-1\right]$

$\frac{\partial l}{\partial \lambda}=-\frac{2 \alpha}{3} \sum_{i=1}^{n}\left[w_{i}^{3}(\beta) w_{i}^{-1}(\underline{\Phi})\right]$

By solving the last three non-linear equations simultaneously, then $\underline{\Phi}=(\hat{\alpha}, \hat{\lambda}, \hat{\beta})$ will be obtained as shown in Section (6).

\section{FISHER'S INFORMATION MATRIX}

The $2^{\text {nd }}$ partial derivatives of the $1^{\text {th }}$ partial derivatives of Eq.(17) with respect to the unknown parameters $(\alpha, \lambda, \beta)$ are given as follows:

$$
\begin{aligned}
& I_{1}=\frac{\partial^{2} l}{\partial \alpha^{2}}=-\left\{\frac{n}{\alpha^{2}}+\frac{4 \lambda^{2}}{9} \sum_{i=1}^{n} w_{i}^{6}(\beta) w_{i}^{-2}(\underline{\Phi})\right\} \\
& I_{2}=\frac{\partial^{2} l}{\partial \alpha \partial \beta}=\frac{2 \lambda}{3} \sum_{i=1}^{n} w_{i}^{2}(\beta) w_{i}^{-1}(\underline{\Phi})\left[3+2 \alpha \lambda w_{i}^{3}(\beta) w_{i}^{-1}(\underline{\Phi})\right] \\
& I_{3}=\frac{\partial^{2} l}{\partial \alpha \partial \lambda}=\frac{-2}{3} \sum_{i=1}^{n} w_{i}^{3}(\beta) w_{i}^{-1}(\underline{\Phi})\left[1+\frac{2 \alpha \lambda}{3} w_{i}^{3}(\beta) w_{i}^{-1}(\underline{\Phi})\right] \\
& I_{4}=\frac{\partial^{2} l}{\partial \beta^{2}}=-2 \sum_{i=1}^{n} w_{i}^{-2}(\beta)\left\{2 \alpha \lambda w_{i}^{3}(\beta) w_{i}^{-1}(\underline{\Phi})[1\right. \\
& I_{5}=\frac{\partial^{2} l}{\partial \beta \partial \lambda}=2 \alpha \sum_{i=1}^{n} w_{i}^{2}(\beta) w_{i}^{-1}(\underline{\Phi})\left[1+\frac{2 \alpha \lambda}{3} w_{i}^{3}(\beta) w_{i}^{-1}(\underline{\Phi})\right] \\
& \left.I_{6}=\frac{\partial^{2} l}{\partial \lambda^{2}}=-\frac{4 \alpha^{2}}{9} \sum_{i=1}^{n}\left[w_{i}^{6}(\beta) w_{i}^{-1}(\underline{\Phi})\right]+1\right\}
\end{aligned}
$$

Therefore, the Fisher's information matrix $(I)$, is obtained as follows: 


$$
I=-\left[\begin{array}{lll}
I_{1} & I_{2} & I_{3} \\
I_{2} & I_{4} & I_{5} \\
I_{3} & I_{5} & I_{6}
\end{array}\right]
$$

The approximate $100(1-\gamma) \%$ confidence intervals $(\boldsymbol{C} . \boldsymbol{I})$ for the unknown parameters $(\alpha, \lambda, \beta)$ are given by: $\mathrm{B}<\underline{\widehat{\Phi}}<$ $A$, where $A=\underline{\widehat{\Phi}}+\mathrm{Z}_{\frac{\gamma}{2}} \sqrt{\operatorname{var}(\underline{\Phi})}, \quad B=\underline{\widehat{\Phi}}-\mathrm{Z}_{\frac{\gamma}{2}} \sqrt{\operatorname{var}(\underline{\Phi})}$

\section{APPLICATION OF $L Q$ DISTRIBUTION}

The estimators and the corresponding summary statistics are obtained by the proposed model using MathCAD program. For a given samples with different choices of $a, \mathrm{~b}$ and $\lambda$ the maximum likelihood estimators (MLEs), the mean squared error (MSE), relative absolute bias (RAB) and the confidence interval are obtained, Table 1, summarizes the results.

Table 4. Estimates the Unknown Parameters with Corresponding Summary Statistics

\begin{tabular}{|c|c|c|c|c|c|c|c|c|c|}
\hline \multicolumn{4}{|c|}{ Initial values } & MLEs & $M S E$ & $R A B$ & variance & $\begin{array}{l}\text { Lower } \\
\text { limit }\end{array}$ & $\begin{array}{l}\text { Upper } \\
\text { Limit }\end{array}$ \\
\hline $\mathrm{n}$ & 11 & $\alpha$ & 0.0492 & 0.0497 & $0.4 \mathrm{E}-6$ & 0.0006 & 0.0001 & 0.0496 & 0.0499 \\
\hline $\mathrm{a}$ & 1.25 & $\beta$ & 4.375 & 3.9268 & 0.2009 & -0.4482 & 0.0386 & 3.8511 & 4.0025 \\
\hline $\mathrm{b}$ & 7.5 & $\lambda$ & 0.15 & 0.1518 & $0.3 \mathrm{E}-5$ & 0.0018 & 0.0238 & 0.1052 & 0.1984 \\
\hline $\mathrm{n}$ & 20 & $\alpha$ & 0.0492 & 0.0495 & $0.1 \mathrm{E}-6$ & 0.0003 & $0.2 \mathrm{E}-6$ & 0.0494 & 0.0497 \\
\hline $\mathrm{a}$ & 1.25 & $\beta$ & 4.375 & 4.1563 & 0.0479 & -0.2188 & 0.0027 & 4.1509 & 4.1616 \\
\hline $\mathrm{b}$ & 7.5 & $\lambda$ & 0.15 & 0.1509 & $0.9 \mathrm{E}-6$ & 0.0009 & 0.0075 & 0.1362 & 0.1657 \\
\hline $\mathrm{n}$ & 25 & $\alpha$ & 0.0492 & 0.0495 & $0.1 \mathrm{E}-6$ & 0.0003 & $0.2 \mathrm{E}-6$ & 0.0493 & 0.0495 \\
\hline $\mathrm{a}$ & 1.25 & $\beta$ & 4.375 & 4.1563 & 0.0479 & -0.2188 & 0.0033 & 4.1497 & 4.1628 \\
\hline $\mathrm{b}$ & 7.5 & $\lambda$ & 0.15 & 0.1510 & $0.1 \mathrm{E}-5$ & 0.0010 & 0.0207 & 0.1105 & 0.1915 \\
\hline $\mathrm{n}$ & 30 & $\alpha$ & 0.0529 & 0.0528 & $0.4 \mathrm{E}-7$ & $-0.6 \mathrm{E}-4$ & $-0.1 \mathrm{E}-5$ & 0.0520 & 0.0529 \\
\hline $\mathrm{a}$ & 1.3 & $\beta$ & 4.35 & 4.3772 & 0.0007 & 0.0272 & 0.0007 & 4.3759 & 4.3785 \\
\hline $\mathrm{b}$ & 7.4 & $\lambda$ & 0.1 & 0.0999 & $0.2 \mathrm{E}-7$ & $-0.9 \mathrm{E}-4$ & 0.0019 & 0.0962 & 0.1036 \\
\hline
\end{tabular}

From Table 4, Estimate the true parameters $\alpha, \beta, \lambda$ well with relatively small MSEs and RAB. Also it is noticed that, the coverage probabilities of the asymptotic confidence interval are close to the nominal level. These results indicate that the proposed model and the asymptotic approximation work well under the situation. Table 5 summarizes the results of some measures of central tendency and dispersion of the $\boldsymbol{L Q}$ distribution for a given samples with different choices of $a, \mathrm{~b}$ and $\lambda$.

Table 5. Some Measures of Central Tendency and Dispersion of the LQ Distribution

\begin{tabular}{|c|c|c|c|c|}
\hline $\mathrm{n}$ & 11 & 20 & 25 & 30 \\
\hline Mean & 3.7243 & 3.9545 & 3.9545 & 4.2466 \\
\hline Median & 5.1490 & 5.1490 & 5.1490 & 5.1490 \\
\hline
\end{tabular}

\begin{tabular}{|c|c|c|c|c|}
\hline Mode & 8.2256 & 8.4718 & 8.4706 & 9.2221 \\
\hline Harmonic mean & 2.1492 & 2.4811 & 2.4815 & 2.9068 \\
\hline Variance & 33.3094 & 33.5757 & 33.5556 & 31.0103 \\
\hline Kurtosis & 0.0343 & 0.0339 & 0.0339 & 0.0370 \\
\hline Skewness & 0.0112 & 0.0110 & 0.0110 & 0.0077 \\
\hline Pearson1 & -0.7799 & -0.7796 & -0.7796 & -0.8935 \\
\hline Pearson2 & -2.3398 & -2.3388 & -2.3389 & -2.6805 \\
\hline
\end{tabular}

Also, Table 6 summarizes the results of the $3^{\text {th }}$ non central moment about zero at different values of the sample size distribution.

Table 6. The $3^{\text {th }}$ non central moment about zero at different values of $\mathbf{n}$

\begin{tabular}{|l|l|l|l|l|l|}
\hline $\mathrm{n}$ & $\mathrm{r}$ & \multicolumn{2}{|c|}{$\dot{\mu}_{r}$} & \multicolumn{1}{c|}{$\mu\left(A_{r}\right)$} & \multicolumn{1}{|c|}{$\mu\left(B_{r}\right)$} \\
\hline \multirow{4}{*}{11} & 1 & $\dot{\mu}_{1}$ & 3.724 & 3.724 & 3.724 \\
\cline { 2 - 6 } & 2 & $\dot{\mu}_{2}$ & 19.642 & 19.642 & 19.642 \\
\cline { 2 - 6 } & 3 & $\dot{\mu}_{3}$ & 118.286 & 118.13 & 118.13 \\
\hline \multirow{4}{*}{20} & 1 & $\dot{\mu}_{1}$ & 3.955 & 3.955 & 3.955 \\
\cline { 2 - 6 } & 2 & $\dot{\mu}_{2}$ & 21.433 & 21.433 & 21.433 \\
\cline { 2 - 6 } & 3 & $\dot{\mu}_{3}$ & 132.73 & 132.574 & 132.574 \\
\hline \multirow{3}{*}{25} & 1 & $\dot{\mu}_{1}$ & 3.9545 & 3.9545 & 3.9545 \\
\cline { 2 - 6 } & 2 & $\dot{\mu}_{2}$ & 21.4305 & 21.4305 & 21.4305 \\
\cline { 2 - 6 } & 3 & $\dot{\mu}_{3}$ & 132.706 & 132.5493 & 132.5493 \\
\hline \multirow{3}{*}{30} & 1 & $\dot{\mu}_{1}$ & 4.2465 & 4.2465 & 4.2465 \\
\cline { 2 - 6 } & 2 & $\dot{\mu}_{2}$ & 23.6018 & 23.6018 & 23.6018 \\
\cline { 2 - 6 } & 3 & $\dot{\mu}_{3}$ & 148.8577 & 148.7605 & 148.7605 \\
\hline
\end{tabular}

\section{CONCLUSION}

In this article, a new model called the LQ distribution was obtained, which extends the UQ distribution. It is observed that the proposed LQ distribution has several desirable properties, such as: expectation, harmonic mean, variance, moments, reliability function, hazard rate function, cumulative hazard function, the moment generating function, the characteristic function, the MLE of the unknown parameter with its variance. Some important related integrations were developed which can be useful for other researches. Therefore, the new LQ distribution to generated data is an extended model to analyze data from complex situations, then it will be important in applied probability, and can be used quite effectively to provide better fits of modeling of symmetric bimodal processes than the UQ distribution. 


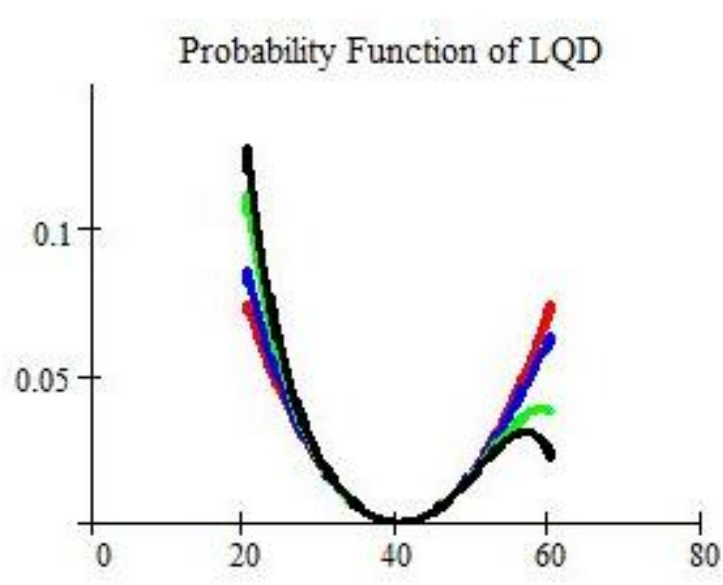

\section{Reliability Function of LQD}

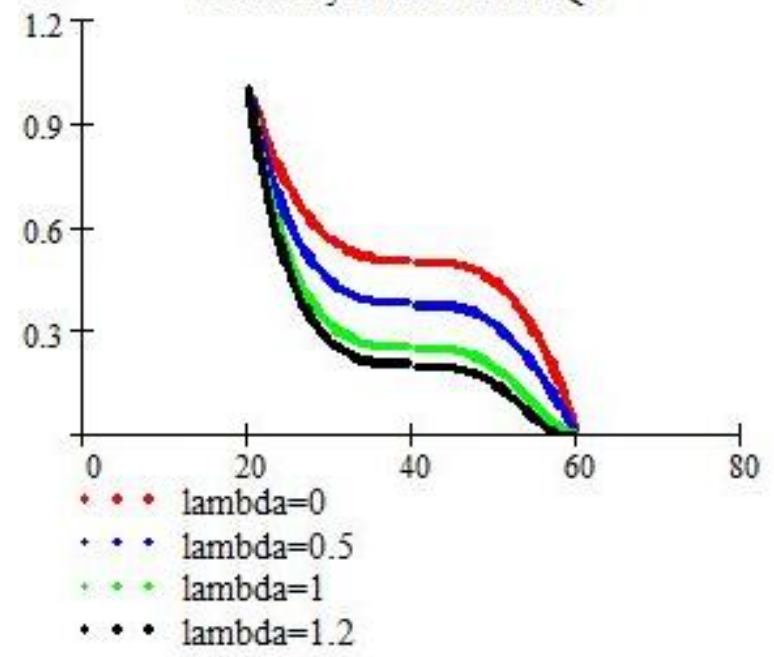

\section{Distribution Function of LQD}
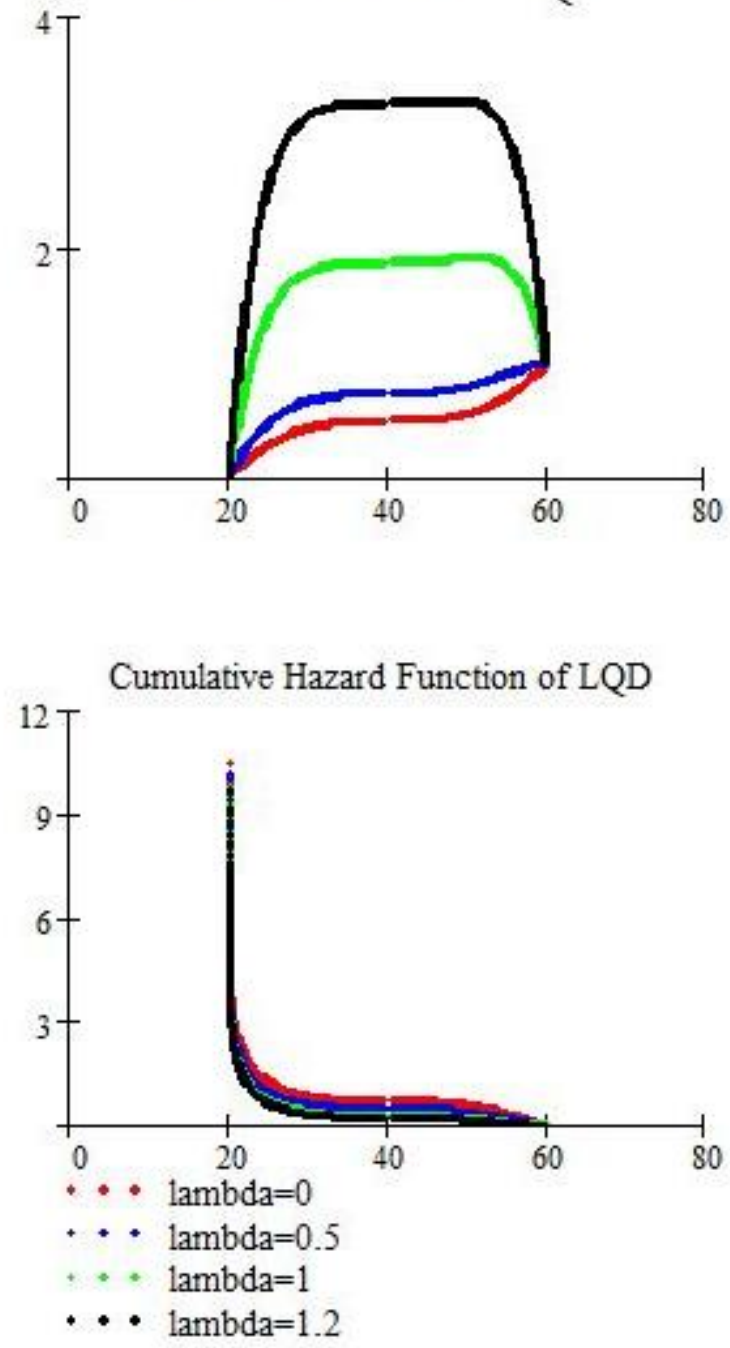

Fig. 1. The $p d f$, $c d f$, reliability and cumulative hazard function of $L Q$ distribution

\section{REFERENCES}

[1] S.O. Edeki, Hilary Okagbuem and Abiodun Opanuga. 2016. The U-quadratic distribution as a proxy for a transformed triangular distribution (TTD). Research Journal of Applied Sciences, 11 (5): 221-223.

[2] Shaw, W. T., \& Buckley, I. R. C. 2007. The alchemy of probability distributions: beyond Gram-Charlier expansions, and a skew-kurtosis normal distribution from a rank transmutation map. arXiv preprint arXiv: 0901.0434 .

[3] Catherine Forbes, Nicholas Hastings, Brian Peacock and Merran Evans. 2011. Statistical Distributions, 4th ed. A John Wiley \& Sons, INC., Publication.

[4] Paul, M., 1970. Introduction to Probability and Statistical Applications. $2^{\text {nd }}$ Edn., Addison-Wesley, Reading, Massachusetts, ISBN. 